\title{
An Unusual Case of Noncatamenial Recurrent Pneumothorax
}

\author{
Andreas Kirschbaum ${ }^{1}$ Timm Greulich ${ }^{2}$ Nikolas Mirow ${ }^{3}$ Detlef K. Bartsch ${ }^{1}$
}

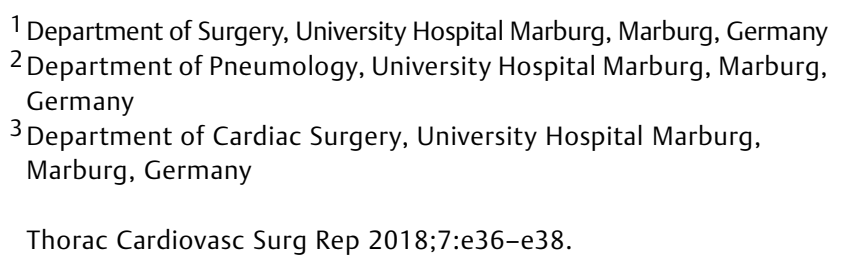

Address for correspondence Andreas Kirschbaum, MD, Department of Surgery, University Hospital Marburg, Baldingerstrasse, Marburg 35033, Germany (e-mail: Kirschbaum001@gmx.de).
Abstract
In a 28-year-old female, seven recurrent pneumothoraces occurred during a period of Keywords 2 years despite multiple thoracic interventions, all on the left thoracic side. Despite
- thoracic surgery profound differential diagnostic analysis, the underlying cause remains unclear. An
- lung
- benign or congenital lesions accumulation of conditions in this patient affecting only the left half of the body is pneumothorax remarkable: sinistral glaucoma as an infant, a sinistral pigmentation disorder, and a sinistral Bochdalek hernia.

\section{Background}

Primary spontaneous and recurrent pneumothoraces occur more frequently in younger people. ${ }^{1}$ It is characteristic that no underlying pulmonary diseases are the cause. In many cases, younger women in particular have catamenial pneumothoraces. ${ }^{2,3}$ Thoracoscopy often reveals small, perforated subpleural blisters on the apex of the lung as supposed morphological cause. They are resected nonanatomically by stapler and always supplemented by subtotal pleurectomy. ${ }^{1}$ After this procedure, the recurrence rate is less than $5 \%$. We present a case in which despite multiple interventions a pneumothorax reoccurred.

\section{Case Report}

A 28-year-old female in good general and nutritional condition (height: $160 \mathrm{~cm}$, weight: $57 \mathrm{~kg}$ ) presented at our hospital with the 5th recurrence of a spontaneous sinistral pneumothorax. Drainage treatment of the initial event and laparoscopy to address free intra-abdominal air had been performed 9 months ago at another hospital. Laparoscopy had shown no intraabdominal abnormalities emphasizing that there were no signs of pelvic endometriosis. Three months later, free intra-abdom- inal air reappeared beneath the left diaphragm, with a sinistral pneumothorax. Due to the patient's history and complete absence of abdominal complaints, another laparoscopy was waived. However, a diagnostic thoracoscopy was performed, and it identified a sinistral Bochdalek hernia, which was closed by a suture. After another 3 months, a sinistral pneumothorax reoccurred, and thoracoscopy and subtotal pleurectomy were performed. Histologically, the parietal pleura was without pathological findings. Two sinistral pneumothoraces occurred at intervals of 3 months, which were drained. After yet another interval of 3 months, sinistral pneumothorax reoccurred for the 5th time and the patient presented at our hospital for the first time. Computed tomography (CT) scan revealed a more strongly chambered pneumothorax, but no evidence of changes in the pulmonary parenchyma (see - Fig. 1). Based on the patient's previous history, we opted for a surgical approach once more. A muscle-saving sinistral thoracotomy was performed on the following day. Except for an adhesion band from the apex of the lung to the thoracic wall, the entire lung surface showed no abnormalities. After the intraoperative venting of the left lung, no air leaked. The lung surface was carefully inspected, but no abnormalities were observed. The Bochdalek hernia-previously described-was sealed, and no other openings were detected. We performed a nonanatomical
License terms Stuttgart - New York

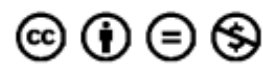

received
March 21, 2018

accepted

June 19, 2018
DOI https://doi.org/

10.1055/s-0038-1667318. ISSN 2194-7635. 


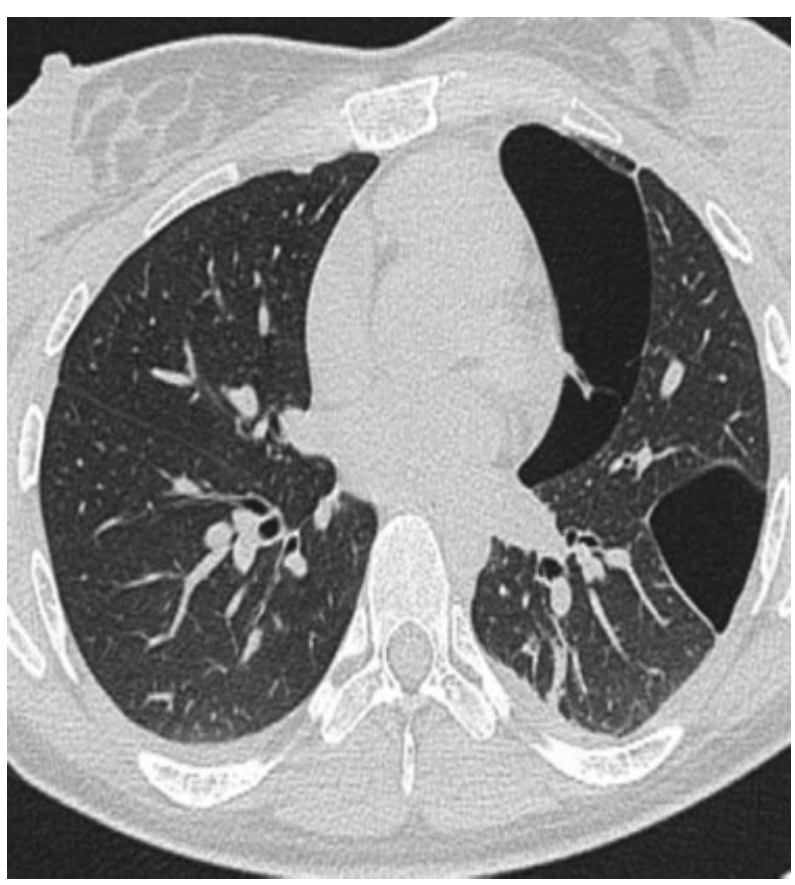

Fig. 1 Computer tomography of the thorax, showing a chambered sinistral pneumothorax; the lung parenchyma shows no abnormalities.

resection of the area described above. In addition, a basal pleurectomy of the residues and coagulation of the entire pleural cavity with an argon beamer were performed. Histological work-up of the samples revealed low-degree subpleural emphysema, without blistering, no evidence of systemic lung disease, or inflammatory changes (see - Fig. 2).

The parietal pleura was likewise unremarkable. The postoperative course was uncomplicated, and the patient was discharged on the 5th postoperative day. Both serologically and genotypically, $\alpha$-antitrypsin deficiency could be excluded. An ultrasound examination of the abdomen also yielded unremarkable findings. The patient had taken oral contraceptives for many years and the incidents were not associated with the patient's menstruatory cycle. Gynecological examination was without pathological findings. Approximately

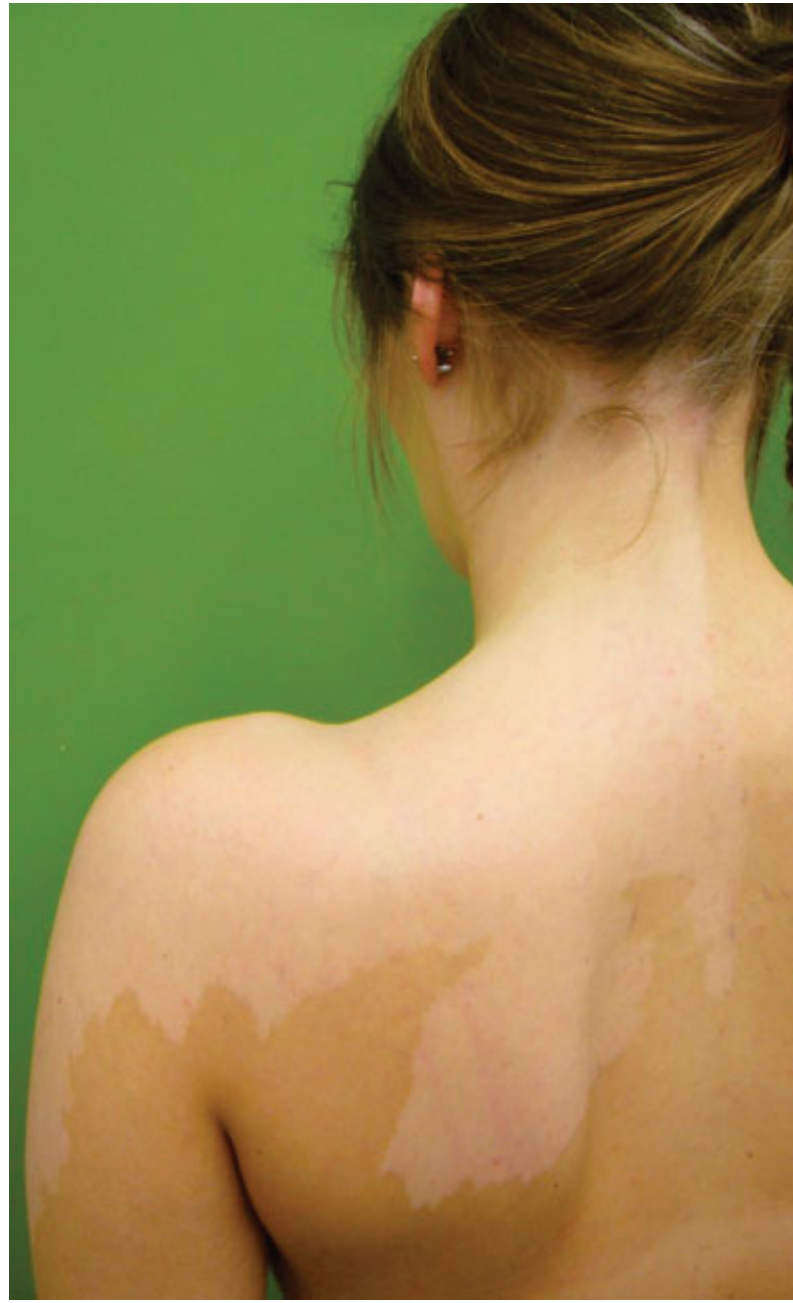

Fig. 3 Congenital segmental pigmentation disorder of the patient (dorsal view).

3 months later, the patient presented again with a sinistral basal pneumothorax. A thoracic drainage was inserted under CT control, followed by a blood pleurodesis ( $50 \mathrm{~mL}$ of autologous blood) 2 days later. Fortunately, the patient has been relapse-free since then. The patient's history of conditions affecting only the left-hand side of the body is noticeable:

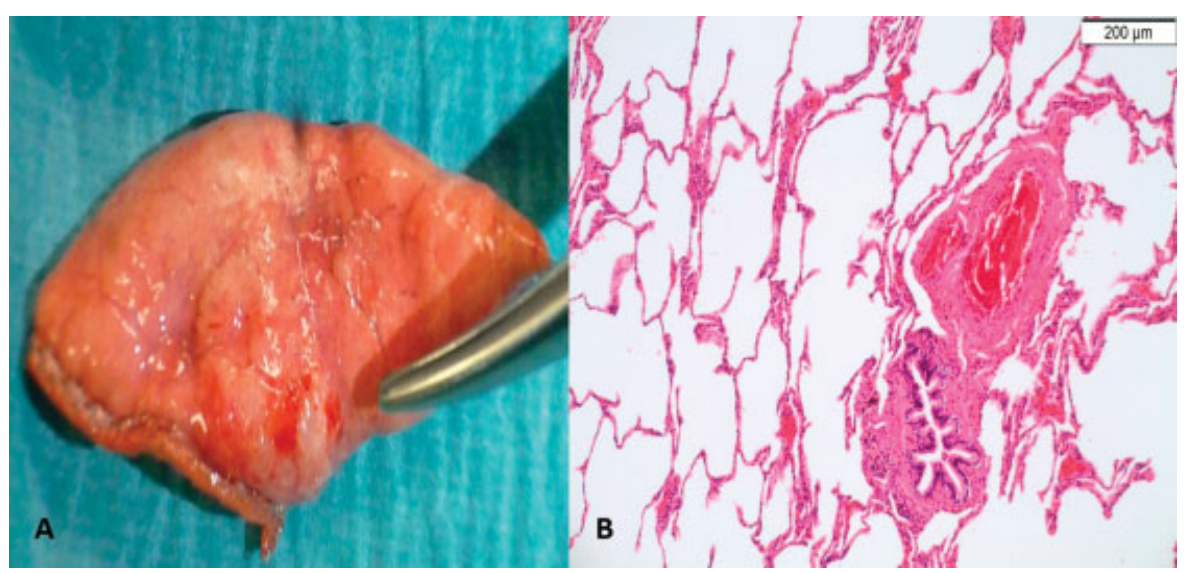

Fig. 2 (A) View of the nonanatomical pulmonary resection sample taken during the surgery; the conspicuous part of the adhesion band is being demonstrated with the instrument. (B) Histological examination of the pulmonary tissue: Normal findings (hematoxylin staining, magnification $100 \times$ ). 
Glaucoma in infancy and a congenital segmental pigmentation disorder sinistrally (see - Fig. 3), as well as a Bochdalek hernia.

\section{Discussion}

In our 28-year-old female patient, spontaneous sinistral pneumothoraces occurred several times within the past 2 years. She is in normal general and nutritional condition, and she never smoked. Several disorders, i.e., glaucoma and vitiligo were observed exclusively on the patients left side even at a very early age. Rogosić et $\mathrm{al}^{4}$ observed concomitant occurrence of glaucoma in vitiligo patients. In 42 patients with vitiligo, during a 2-year period glaucoma was diagnosed in $57 \%$. In none of these patients Rogosić et al additionally described pneumothorax. In our patient due to the normal pulmonary parenchyma in CT scans, as well as the normal histological findings, the following diseases are excluded as the cause: BirtHogg-Dubé syndrome, Langerhans cell histiocytosis, and lymphangioleiomyomatosis. There was nothing to suggest a connective tissue disease either, given the normal skin texture and mobility of the joints. The patient's apparent physique looked quite normal too. Sonography of the abdomen with respect to the aorta and kidneys yielded unremarkable findings.

An $\alpha$-1-antitrypsin deficiency could be excluded serologically and genotypically. Histologically, a small subpleural emphysema without typical bullous changes was described. The likely cause of the pneumothoraces could therefore be minute subpleural vesicles. This is supported by the fact that after insertion of each thoracic drainage, the respective bronchopleural fistulae immediately stopped, indicating that the parenchymal leak probably was not very large.

Lillington first described catamenial pneumothorax in $1972 .^{5}$ The term is used for recurrent pneumothorax 72 hours before or after and within 5 to 7 days after onset of menstruation. In our case, occurrence of the pneumothoraces was in no relation to the menstruatory cycle. In most cases of catamenial pneumothoraces, the right side is affected. What hinted at catamenial pneumothorax, however, is the fact that in the first two pneumothoraces free intra-abdominal air was diagnosed. In addition, a diaphragmatic defect was diagnosed in terms of a Bochdalek hernia. Typically, in catamenial pneumothorax, single or multiple defects appear in the central portion of the diaphragm. Regional endometriosis is often found nearby. ${ }^{5}$

Cases of endometriosis affecting exclusively the pleurae have also been described in the literature. Thoracic pain connected to the menstruatory cycle, recurrent hemorrhagic pleural effusions, and spontaneous pneumothoraces are typical. Contrary to our case, symptoms are mainly right sided. ${ }^{6}$ Several causes have been postulated as causes of catamenial pneumothorax. During menstruation, air may rise through the tubes and enter the pleural space via preexisting diaphragmatic defects. As another cause, an increase in prostaglandin F2 levels is being discussed, which may lead to broncho - and vasoconstriction resulting in ruptured subpleural blisters. The presence of thoracic endo- metrial lesions is thought to be caused by metastatic seeding of endometrial cells via uterine veins. ${ }^{5}$ In our case, the cause of the free intra-abdominal air remained unknown, there was no clinical or histological evidence of thoracic endometriosis, or other connection to menstruation.

As can be seen from our case, the treatment of pneumothorax can be extremely tedious and distressing for those affected. It may be critically noted that surgical treatment of the first recurrent pneumothorax should have been performed more extensively. Duplication or coverage of the diaphragm would have been a good addition to the occlusion of the Bochdalek hernia. In the literature, the diaphragm has been reportedly covered with polyglactin, polypropylene mesh, polytetrafluoroethylene mesh, or bovine pericardium. ${ }^{7}$ Certainly, at that time total removal of the parietal pleura would have been indicated. It is difficult to accept that this was omitted.

During the open reoperation that we performed, the diaphragmatic surface was closely inspected and no abnormalities were observed, therefore we performed thermal coagulation only.

If the affected lung cannot be bonded to the thoracic wall by total pleurectomy, a reoccurring pneumothorax is usually treated by thoracic drainage. CT-controlled insertion may prove useful in these cases. After reexpansion of the lung, we recommend additional blood pleurodesis. In our judgment, repeated surgical explorations are not promising. In consensus with gynecologists consulted, due to the lacking evidence of a catamenial context in our patient, we abstained from administrating gonadotropin hormones.

Unfortunately, in our patient further recurrences of pneumothorax may be expected.

\section{Summary}

We report the case of a recurrent sinistral pneumothorax, which keeps on reoccurring despite repeated treatment. Its underlying cause is unclear but surely noncatamenial.

\section{References}

1 Ghezel-Ahmadi D, Bölükbas S, Fischer T, Ghezel-Ahmadi V, Schirren J. Pneumothorax, what kind of therapy is necessary? A clinical overview [in German]. Zentralbl Chir 2012;137(03):214-222

2 Alifano M, Legras A, Rousset-Jablonski C, et al. Pneumothorax recurrence after surgery in women: clinicopathologic characteristics and management. Ann Thorac Surg 2011;92(01):322-326

3 Nakamura H, Konishiike J, Sugamura A, Takeno Y. Epidemiology of spontaneous pneumothorax in women. Chest 1986;89(03):378-382

4 Rogosić V, Bojić L, Puizina-Ivić N, et al. Vitiligo and glaucoma - an association or a coincidence? A pilot study. Acta Dermatovenerol Croat 2010;18(01):21-26

5 Visouli AN, Zarogoulidis K, Kougioumtzi I, et al. Catamenial pneumothorax. J Thorac Dis 2014;6(Suppl 4):S448-S460

6 Gräbner D, Seidel G, Weber J. Endometriosis externa thoracalis [in German]. Zentralbl Chir 1988;113(02):101-107

7 Marjański T, Sowa K, Czapla A, Rzyman W. Catamenial pneumothorax - a review of the literature. Kardiochir Torakochirurgia Pol 2016;13(02):117-121 